\title{
Des modes de capture du carbone et de la compétitivité relative des énergies primaires
}

\author{
Jean-Pierre Amigues ${ }^{1}$, Gilles Lafforgue ${ }^{2}$ et Michel Moreaux ${ }^{3}$
}

17 avril 2013

\begin{abstract}
Résumé
Nous caractérisons les sentiers d'exploitation optimale de deux énergies primaires parfaitement substituables: une ressource non renouvelable carbonée et une ressource renouvelable non polluante. Ces ressources approvisionnent les besoins en énergie de deux secteurs qui se différencient par leur accès à des technologies d'abattement différentes. Le secteur 1 peut avoir recours au captage et stockage du carbone (CSC) à un coût raisonnable. Le secteur 2 n'a accès qu'à la technologie de capture atmosphérique nettement plus coûteuse. Avec une structure de coûts constants et en supposant que le stock de pollution ne peut dépasser un certain seuil critique, nous montrons qu'il est optimal de commencer à capturer les émissions du secteur 1 avant que ce seuil ne soit atteint et que cette capture doit, dans un premier temps, porter sur la totalité des rejets de ce secteur. La capture des émissions du secteur 2 n'intervient qu'une fois le plafond de pollution atteint et n'est que partielle. Enfin, la taxe carbone optimale est croissante durant la phase avant plafond, puis décroît par paliers jusqu'à 0 .
\end{abstract}

Mots clefs : Changement climatique ; Energie ; CSC ; Capture atmosphérique ; Taxe carbone. Classification JEL : Q32, Q42, Q54, Q58.

\begin{abstract}
We characterize the optimal exploitation paths of two perfect substitute primary energy resources, a non-renewable polluting resource and a carbon-free renewable one. Both resources can supply the energy needs of two sectors. Sector 1 is able to reduce its carbon footprint at a reasonable cost owing to a CCS device. Sector 2 has only access to the air capture technology, but at a significantly higher cost. We assume that the atmospheric carbon stock cannot exceed some given ceiling. We show that it is optimal to begin by fully capturing the sector 1's emissions before the ceiling is reached and next, to deploy the air capture in order to partially abate sector 2's emissions. The optimal carbon tax is first increasing during the pre-ceiling phase and next, it declines in stages down to 0 .
\end{abstract}

Keywords: Climate change; Energy; CCS; Air capture; Carbon tax.

\footnotetext{
${ }^{1}$ Toulouse School of Economics (INRA-LERNA).

${ }^{2}$ Auteur correspondant. University of Toulouse, Toulouse Business School. 20 Bd Lascrosses - BP $7010-31068$ Toulouse Cedex 7, France. Email : g.lafforgue@esc-toulouse.fr. Les auteurs remercient le Conseil Français de I'Energie pour son soutien financier.

${ }^{3}$ Toulouse School of Economics (IDEI-LERNA).
} 


\section{Introduction}

Les ressources carbonées fossiles sont des énergies primaires abondantes dont la mobilisation permet de satisfaire les besoins en services énergétiques des usagers à des coûts relativement modestes. Leur compétitivité par rapport à d'autres ressources primaires, en particulier les énergies renouvelables, semble donc assurée. Cette perspective de développement risque cependant d'être compromise par les rejets conséquents de gaz à effet de serre (GES) qu'implique leur utilisation massive, gaz dont l'accumulation dans l'atmosphère, dès lors qu'elle est trop forte, peut déclencher des dommages difficilement supportables.

Pour contourner un handicap susceptible de s'avérer à terme dirimant, un facteur clé est la possibilité de maîtriser à coûts raisonnables les rejets de GES qu'implique l'exploitation soutenue de ces ressources fossiles. L'un des moyens envisagés pour réduire les rejets de $\mathrm{CO}_{2}$ dans l'atmosphère est le captage et la séquestration géologique du carbone (CSC), solution notamment préconisée par le GIEC (Groupe d'Experts Intergouvernemental sur I'Evolution du Climat) dans un rapport technique dédié (IPCC, 2005). Sans entrer dans les détails techniques, qui font par ailleurs l'objet d'une abondante littérature spécialisée, ce procédé d'abattement consiste à capter à la source les émissions de composés carbonées avant rejet dans l'atmosphère et à les injecter ensuite dans des réservoirs naturels (par exemple des aquifères salins), ou d'anciens sites miniers ou encore dans des gisements $d^{\prime}$ hydrocarbures (en cours d'exploitation ou épuisés) ${ }^{4}$. Les estimations actuelles suggèrent que cette technologie est une solution crédible en termes de coût-efficacité pour éliminer une grande partie (de 80 à $90 \%$ selon le GIEC) des émissions provenant des centrales thermiques au charbon ou à gaz. Islegen et Reichelstein (2009) soulignent en outre que ce coût est " raisonnable " compte tenu du coût social du carbone auquel il doit être comparé. L'Agence Internationale de l'Energie estime pour sa part que le prix de la tonne de $\mathrm{CO}_{2}$ (reflété par une taxe ou un marché de droits) à partir duquel il devient rentable de déployer le CSC se situe entre 30 et $45 \$$ pour les centrales thermiques à charbon et aux alentours de $60 \$$ pour les centrales à gaz (IEA, 2006). Ce niveau de prix devrait baisser à $25 \$ / \mathrm{tCO}_{2}$ à I'horizon 2030 compte tenu des évolutions technologiques ${ }^{5}$.

Cependant, malgré son fort potentiel, le CSC présente l'inconvénient de ne pouvoir être mis en œuvre à des coûts raisonnables que pour les rejets de $\mathrm{CO}_{2}$ qui émanent d'une partie seulement des utilisateurs d'énergie : ceux qui produisent les émissions les plus importantes et les plus concentrées, typiquement les centrales électriques thermiques ou certaines industries lourdes (cimenteries, aciéries...). En revanche, cette technologie s'avère

\footnotetext{
${ }^{4}$ Le plus ancien dispositif de CSC encore actif, le projet Sleipner, est situé en Mer du Nord (Finlande). La plus grande installation au monde est le projet Weyburn-Midale, situé au Canada. En France, l'ancien gisement gazier de Laque, dans les Pyrénées Atlantiques, fait office de site d'expérimentation du CSC pour l'entreprise Total. Cependant, comme le remarque Herzog (2011), cette technologie a été développée à l'origine pour un tout autre objectif que la réduction des émissions carbonées. Le premier dispositif industriel de CSC, construit aux Etats-Unis à la fin des années 70, avait pour but d'améliorer la productivité des gisements pétroliers en cours d'exploitation. Le $\mathrm{CO}_{2}$ capté après combustion était alors réinjecté dans le puits pour y augmenter la pression et faciliter ainsi l'extraction (" enhanced oil recovery operations »).

${ }^{5}$ Pour plus de détails sur les estimations de coûts, de capacité et de développement, nous renvoyons le lecteur aux rapports de l'AIE (2006) et du MIT (2007). Hamilton et al. (2009) et Herzog (2011) font également un état des lieux assez complet des possibilités qu'offre le CSC, mais également de ses limites.
} 
inappropriée, ou alors infiniment coûteuse, pour traiter les émissions de plus faible ampleur ou plus dispersées comme celles provenant du secteur des transports ou des activités agricoles par exemple. II semble en effet évident que capter les rejets d'une centrale thermique à gaz sera moins coûteux que capter les rejets d'un parc de véhicules fonctionnant grâce à cette même source d'énergie. Toutefois, si la capture directe apparaît irréalisable dans certains cas, il reste la possibilité d'une capture indirecte en prélevant dans l'atmosphère les gaz qui y auraient été rejetés. L'extension du couvert végétal est un des procédés possibles, mais limité. Certaines voies industrielles semblent s'ouvrir à terme qui ne rencontreraient pas ces limites, comme par exemple la capture atmosphérique par voie chimique ${ }^{6}$. Un tel procédé permet de capter tous les types d'émissions, diffus ou concentrés, mais s'avère beaucoup plus coûteux que le CSC standard comme le soulignent Barrett (2009) et Keith (2009). Selon ce dernier, le coût marginal de la capture chimique est estimé entre 100 et $200 \$ / \mathrm{tCO}_{2}$, niveau largement supérieur au coût du CSC et au coût social du carbone (compris entre 7 et $85 \$ / \mathrm{tCO}_{2}$ toujours selon ce même auteur). Cependant, McKay (2009) conclut que, même si les besoins en énergie nécessaires pour mener à bien une telle opération sont pour l'instant énormes, il convient pourtant de considérer très sérieusement la capture atmosphérique car elle peut s'avérer en fin de compte la dernière ligne de défense contre le changement climatique si l'humanité échoue dans sa recherche de procédés d'abattement moins coûteux.

Cet article aborde la question de cette différence d'accès aux technologies de capture selon les secteurs d'utilisation de l'énergie. En particulier, son objectif est de montrer en quoi cette hétérogénéité peut modifier les trajectoires optimales d'utilisation des différentes énergies primaires, de séquestration des émissions et du coût social de la pollution.

Le modèle sur lequel nous nous fondons est issu des travaux de Lafforgue et al. (2008-a et 2008-b), qui constituent eux-mêmes une extension du modèle séminal de Chakravorty et al. (2006). Deux types d'énergies primaires, parfait substitut l'une de l'autre, sont disponibles: une ressource non-renouvelable polluante, le charbon, et une ressource renouvelable non carbonée, l'énergie solaire. Ces deux sources peuvent approvisionner la demande en services énergétiques émanant de deux secteurs qui se distinguent par leur accès aux différents modes d'abattement. Le secteur 1, l'industrie lourde ou les centrales électriques thermiques par exemple, a accès au CSC et peut ainsi réduire ses émissions à un coût raisonnable. Le secteur 2 , le secteur des transports par exemple, n'a accès qu'à la capture atmosphérique dont le coût est significativement plus élevé que celui du CSC.

Pour aller à l'essentiel, nous retenons comme modèle des dommages le modèle dit " à plafond ", introduit par Chakravorty et al. (2006), qui consiste à poser que les dommages sont négligeables tant qu'un seuil critique de concentration atmosphérique n'est pas dépassé mais sont incommensurablement élevés dès lors que ce seuil est franchi. Ainsi, la concentration atmosphérique en carbone est contrainte de ne pas dépasser ce seuil, ce qui

\footnotetext{
${ }^{6}$ Cette technique consiste à récupérer le $\mathrm{CO}_{2}$ directement dans l'atmosphère par voie chimique en utilisant un liquide alkalin pour en isoler les molécules. Le $\mathrm{CO}_{2}$ est ensuite acheminé et séquestré dans des réservoirs selon des procédés proches de ceux du CSC. D'autres voies expérimentales existent, comme la stimulation des microalgues océaniques ou la pulvérisation de roches absorbantes dans l'atmosphère. Mais aucune n'offre pour l'instant de débouchés suffisamment crédibles pour être envisagée à échelle industrielle (Barrett, 2009). Notons toutefois que la solution la plus naturelle, mais non abordée dans cet article, consiste simplement à exploiter le processus de la photosynthèse, par exemple en augmentant les surfaces boisées ou en changeant les pratiques agricoles culturales.
} 
est l'objectif déclaré de l'accord de Kyoto. Dans ce type de modèle, puisque les ressources carbonées fossiles sont disponibles en grande quantité et d'un coût de mobilisation relativement modeste, si le seuil de déclenchement d'évènements catastrophiques n'est pas excessivement élevé, la contrainte de non-franchissement du dit seuil sera nécessairement active le long du sentier optimal d'exploitation des ressources polluantes ${ }^{7}$. Deux points méritent alors d'être soulignés. Le premier est le fait que, le long d'un sentier optimal, la date à partir de laquelle la contrainte de plafond restreint la consommation de charbon est une date phare. Etant fonction du sentier de consommation de ce type d'énergie suivi depuis l'instant initial, elle est de ce fait endogène. La contrainte de plafond doit donc faire sentir ses effets sur la totalité du sentier d'exploitation du charbon, mais aussi sur celui de l'énergie solaire puisque ces deux sources d'énergie sont de parfaits substituts l'une de l'autre.

Le second point à souligner est que la société dispose de deux options pour relâcher cette contrainte de plafond, ces deux options pouvant être combinées. L'une consiste à recourir aux moyens de capture et de séquestration, l'autre à substituer la ressource renouvelable à la ressource fossile. De ce fait, le problème est double. i) Pour déverrouiller la contrainte, à supposer qu'il faille la déverrouiller, faut-il privilégier la capture des rejets émis par la ressource polluante et retarder l'exploitation de la ressource naturellement propre ? Ou faut-il au contraire privilégier d'abord l'exploitation de la ressource naturelle dite propre ? ii) Quelle que soit la réponse à cette question, faut-il attendre d'être contraint pour mobiliser l'une ou l'autre de ces deux sortes de ressources, naturellement propre ou rendue propre après traitement approprié, ou faut-il au contraire s'efforcer de les mettre en œuvre avant d'avoir à subir directement les effets de la contrainte comme le suggèrerait une acceptation large du principe de précaution ? Clairement, les réponses à ces deux questions sont liées. L'argumentation sur la compétitivité relative de ces options est fondée sur la confrontation de leurs coûts moyens totaux d'utilisation.

Dans cet article, nous restreignons l'analyse aux scenarii dans lesquels le coût de l'énergie solaire est trop élevé pour permettre une utilisation simultanée des deux types d'énergie primaire. Dans ce cas, le poids de la contrainte environnementale ne peut être allégé que par abattement des émissions. Les scenarios dans lesquels l'énergie solaire se substitue partiellement au charbon pour relâcher la contrainte sont étudiés dans un papier "compagnon" (Amigues et al., 2012-b). Nous montrons alors que dans le scenario impliquant la mobilisation des deux modes de capture, il est optimal de commencer à séquestrer les émissions du secteur 1 avant l'atteinte du plafond. De plus, cette capture s'applique, tout au moins au début, à la totalité des émissions de ce secteur. Ces deux premiers résultats contrastent avec ceux obtenus dans Chakravorty et al. (2006) et Lafforgue et al. (2008-a) et résultent directement du fait que le CSC, la technologie d'abattement la moins coûteuse, ne peut traiter la totalité des rejets émis par l'économie, mais au maximum ceux du secteur 1. Enfin, nous montrons qu'il est optimal de déployer la capture atmosphérique pour abattre une partie des émissions du secteur 2 qu'une fois le plafond atteint.

\footnotetext{
${ }^{7}$ On peut démontrer que la prise en compte de dommages relativement mineurs pour des niveaux de pollution atmosphérique inférieurs à ce seuil critique ne modifie pas les principales propriétés qualitatives des sentiers optimaux. Sur ce point, voir Amigues et al. (2011).
} 
L'article est organisé comme suit. La Section 2 présente les hypothèses du modèle. Dans la Section 3, nous définissons le programme du planificateur social et dérivons les conditions d'optimalité. Celles-ci sont ensuite commentées de façon à identifier l'ensemble des arbitrages de choix de consommation énergétique et d'option d'abattement. Dans la Section 4, nous caractérisons le sentier optimal pour lequel les deux modes de capture sont déployés. La Section 5 propose une analyse du profil temporel de la taxe carbone optimale et du poids total de l'externalité environnementale supporté par chaque secteur. Enfin, nous concluons dans la Section 6.

\section{Présentation du modèle}

La structure du modèle est représentée par la Figure 1.

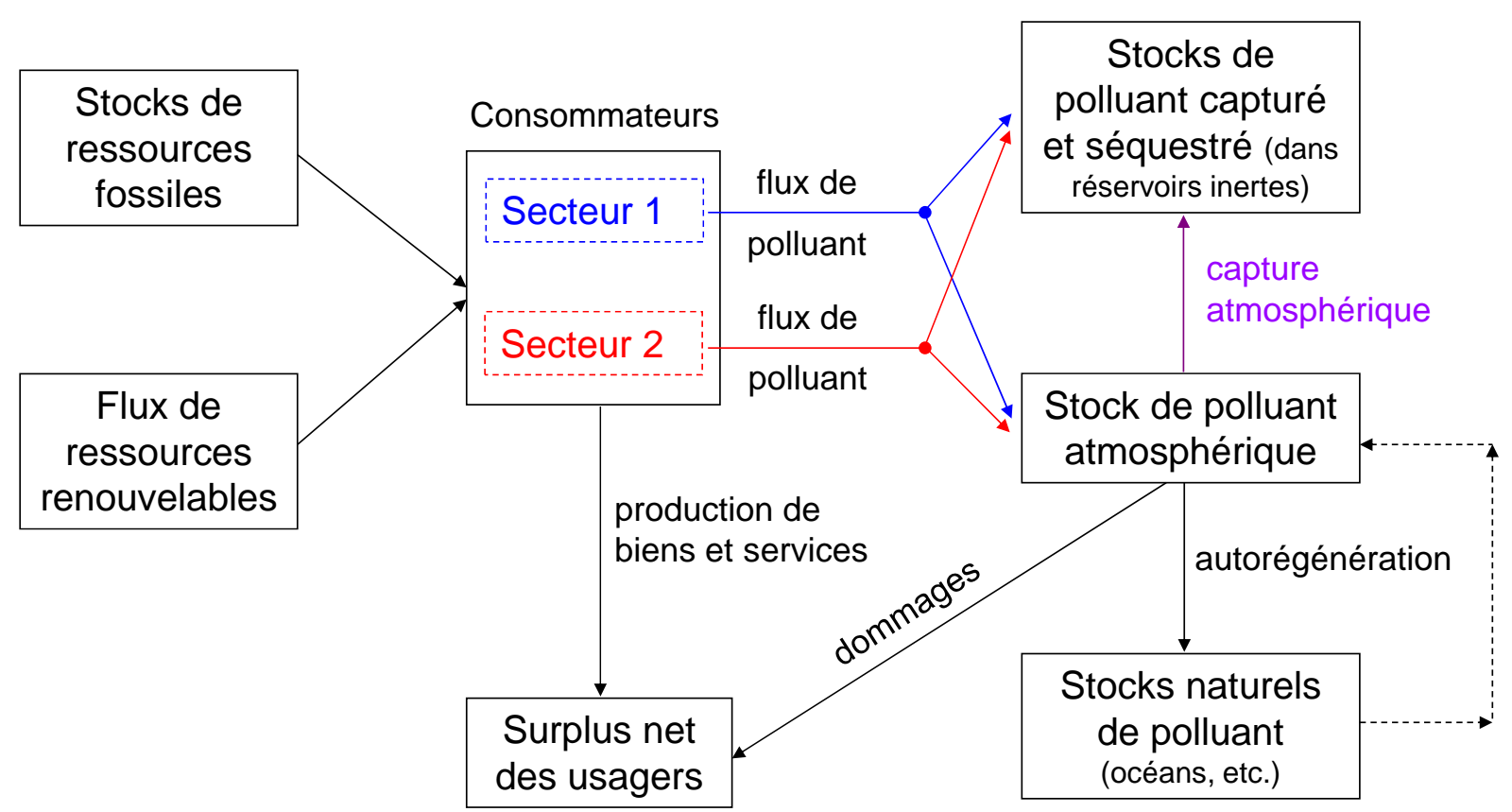

Figure 1. Structure du modèle

Demandes sectorielles :

On considère une économie dans laquelle deux secteurs, indicés par $i=1,2$, consomment des services énergétiques. Le surplus brut instantané $u\left(q_{i}\right)$ que l'usager de type $i$ retire de la consommation de $q_{i}$ unités d'énergie finale est supposé identique dans chaque secteur ${ }^{8}$. Cette fonction vérifie les conditions standards: elle est de classe $\mathcal{C}^{2}$,

\footnotetext{
${ }^{8}$ Coulomb et Henriet (2010) étudient le cas de demandes sectorielles distinctes qui présentent des différences d'échelle dans un modèle à deux secteurs et une seule technologie d'abattement. Comparé à nos résultats, I'incidence d'une telle hypothèse est très faible. Aussi supposons-nous une fonction de demande similaire pour les deux secteurs afin d'axer l'analyse sur les différences dans les technologies d'abattement auxquelles ont accès ces secteurs.
} 
strictement croissante, strictement concave et satisfait la condition d'Inada en 0 , i.e. $\lim _{q \downarrow 0} u^{\prime}\left(q_{i}\right)=+\infty$. On note $p_{i}\left(q_{i}\right)=u^{\prime}\left(q_{i}\right)$ le surplus brut marginal associé, c'est-à-dire le prix de l'énergie au consommateur, et $q_{i}^{d}(p)=p_{i}^{-1}\left(q_{i}\right)$ la fonction de demande sectorielle directe.

\section{Energies primaires :}

La demande en services énergétiques dans chaque secteur peut être approvisionnée par deux ressources primaires, parfait substitut l'une de l'autre : une ressource carbonée fossile et émettrice de $\mathrm{CO}_{2}$, le charbon, et une ressource renouvelable propre, l'énergie solaire. A chaque instant $t$, les flux de consommation de ces deux sources d'énergie par le secteur $i$, sont notés respectivement $x_{i}(t)$ et $y_{i}(t)$. La demande sectorielle finale s'écrit donc $q_{i}(t)=x_{i}(t)+y_{i}(t)$. Puisque, du fait de la condition d'Inada ${ }^{9}, q_{i}(t)$ ne peut être nul excepté asymptotiquement - les flux de consommations sectorielles de charbon et d'énergie solaire doivent vérifier les conditions suivantes :

$$
\begin{aligned}
& x_{i}(t) \geq 0, \quad i=1,2 \\
& y_{i}(t) \geq 0, \quad i=1,2
\end{aligned}
$$

avec $x_{i}(t)+y_{i}(t)>0$. Le coût d'approvisionnement à partir de l'une ou l'autre de ces deux sources primaires, qui comprend l'ensemble des coûts d'acheminement et de transformation de l'énergie primaire en question en services énergétiques directement utilisables, est supposé identique dans chaque secteur. Les coûts marginaux associés, notés respectivement $c_{x}$ et $c_{y}$, sont supposés constants. On pose que le coût marginal direct d'utilisation du charbon est inférieur au coût marginal direct du solaire : $c_{x}<c_{y}$.

Le charbon étant non renouvelable et disponible en quantité limitée, sa consommation doit obéir à un processus d'épuisement, ce qui implique d'imputer une rente de rareté à son utilisation. En notant $X^{0}$ les réserves initiales, la dynamique du stock courant $X(t)$ de charbon (commun aux deux secteurs) est donnée par :

$$
\dot{X}(t)=-\sum_{i} x_{i}(t), \text { avec } X(0) \equiv X^{0} \text { et } X(t) \geq 0
$$

Le substitut solaire est disponible à chaque instant sous la forme d'un flux naturel suffisamment abondant pour satisfaire la demande des deux secteurs. En notant $\tilde{y}$ la consommation sectorielle maximale, solution de l'équation $u^{\prime}(y)=c_{y}$ i.e. $\tilde{y}=q^{d}\left(c_{y}\right)$, ce flux naturel doit donc être supérieur à chaque instant à $2 \tilde{y}$. Par conséquent, aucune rente ne doit être imputée à l'utilisation de l'énergie solaire, contrairement à celle du charbon.

\footnotetext{
${ }^{9}$ Cette condition stipule que l'utilité marginale tend vers l'infini lorsque la consommation tend vers 0 . Consommer une quantité d'énergie, même infinitésimale, procurera donc toujours à la marge un gain en termes d'utilité supérieur à celui obtenu lorsque la consommation est nulle.
} 
On note $Z(t)$ le niveau de concentration atmosphérique de carbone à l'instant $t$, et $Z^{0}$ le niveau initial. L'ampleur des dommages dépend clairement du niveau $Z(t)$. Cependant, plutôt que d'introduire une fonction de dommage continue, nous supposons qu'il existe un certain seuil $\bar{Z}$ de concentration de carbone (identifié par la communauté scientifique) audelà duquel un dommage catastrophique, irréversible et infiniment coûteux pour la société surviendra ${ }^{10}$. Sachant cela, le planificateur intègre cet élément dans son problème de décision en contraignant $Z(t)$ à ne pas dépasser $\bar{Z}$.

L'accroissement du stock atmosphérique est dû aux émissions carbonées générées par la combustion de charbon dans chaque secteur. En notant $\zeta$ le contenu en carbone d'une unité de charbon, le flux total de pollution potentiellement rejeté dans l'atmosphère s'élève à chaque instant à $\zeta \sum_{i} x_{i}(t)$. Cependant, il est possible de réduire ces émissions grâce à deux types de technologies d'abattement : le CSC et la capture atmosphérique. On note respectivement $c_{e}$ et $c_{a}$ le coût marginal de séquestration du carbone selon qu'il est capturé à la source (CSC) ou dans l'atmosphère. Ces deux coûts sont supposés constants ${ }^{11}$ et tels que $c_{e}<c_{a}$. On suppose également que le niveau de ces deux coûts n'est pas trop élevé pour qu'il soit optimal de mobiliser l'une ou l'autre des technologies d'abattement en temps fini. Enfin, quel que soit le mode utilisé, les émissions capturées sont stockées dans des réceptacles dont la capacité est illimitée ${ }^{12}$.

On suppose que les deux secteurs considérés sont hétérogènes et se différencient selon le type de technologie de capture à laquelle ils ont accès. Le secteur 1 peut réduire ses émissions à la source en utilisant le CSC, mais pas le secteur 2. Le seul moyen pour la société de traiter les émissions issues du secteur 2 est d'avoir recours à la capture atmosphérique. On note $s_{e}(t)$ la part des émissions du secteur 1 qui est directement capturée par CSC (les émissions résiduelles de ce secteur sont donc égale à $\left.\zeta x_{1}(t)-s_{e}(t)\right)$ et $s_{a}(t)$ la quantité de carbone qui est directement prélevée dans l'atmosphère. Ces flux doivent vérifier à tout instant les contraintes de non-négativité suivantes :

$$
\begin{aligned}
& \zeta x_{1}(t)-s_{e}(t) \geq 0, \quad s_{e}(t) \geq 0 \\
& s_{a}(t) \geq 0
\end{aligned}
$$

\footnotetext{
${ }^{10}$ Techniquement, cela revient à définir une fonction de dommage $D(Z)$ telle que $D^{\prime}(Z)=0$ pour $Z(t) \leq \bar{Z}$ et $D^{\prime}(Z)=+\infty$ pour $Z(t)>\bar{Z}$ ou, plus généralement, une fonction d'utilité $U(q, Z)$ telle que $U(q, Z)=u(q)$ pour $Z(t) \leq \bar{Z}$ et $U(q, Z)=-\infty$ pour $Z(t)>\bar{Z}$.

${ }^{11}$ Voir Amigues et al. (2012-a) pour des structures non linéaires de coûts d'abattement.

12 La question de la limite de capacité des sites de stockage et de leur dynamique de remplissage est traitée dans Lafforgue et al. (2008-a) et dans Ayong le Kama et al. (2012). On peut montrer que la prise en compte de cette hypothèse ne remettrait pas en cause les principaux résultats obtenus, en particulier l'allure générale du sentier optimal.
} 
Enfin, une partie du $\mathrm{CO}_{2}$ accumulé dans l'atmosphère est progressivement éliminée par auto-régénération naturelle (absorption par les océans ou la biosphère) à un taux constant $\alpha$. En prenant en compte toutes les composantes de la dynamique de $Z(t)$, il en résulte :

$$
\begin{aligned}
& \dot{Z}(t)=\zeta\left[x_{1}(t)+x_{2}(t)\right]-\left[s_{e}(t)+s_{a}(t)\right]-\alpha Z(t), \quad Z(0) \equiv Z^{0}<\bar{Z} \\
& \bar{Z}-Z(t) \geq 0
\end{aligned}
$$

Lorsque le stock atmosphérique de carbone atteint son niveau critique, i.e. $Z(t)=\bar{Z}$, et en l'absence de toute capture des émissions, i.e. $s_{e}(t)=s_{a}(t)=0$, l'équation (6) implique alors que la consommation totale de charbon des deux secteurs est au plus égale à $\bar{x} \equiv \alpha \bar{Z} / \zeta$. Dans ce cas de figure, puisque les deux secteurs ont la même fonction de demande et donc le même poids dans l'économie, chacun utilise une quantité identique de charbon égale à $\bar{x} / 2$.

Bien-être social :

Le surplus net instantané du secteur $i$ - hors coût éventuel de capture -est défini par $S_{i}\left(x_{i}(t), y_{i}(t)\right) \equiv u\left(x_{i}(t)+y_{i}(t)\right)-c_{x} x_{i}(t)-c_{y} y_{i}(t)$. Si la contrainte (7) est satisfaite, la fonction de bien-être social, notée $W$, s'écrit comme la somme actualisée au taux d'escompte social $\rho, \rho>0$, des surplus sectoriels totaux diminuée des éventuelles dépenses de capture :

$$
W=\int_{0}^{\infty}\left[S_{1}(t)+S_{2}(t)-c_{e} s_{e}(t)-c_{a} s_{a}(t)\right] e^{-\rho t} d t
$$

Dans le cas contraire, si le seuil critique de concentration atmosphérique $\bar{Z}$ est dépassé, on a $W=-\infty$.

\section{Programme du planificateur et conditions d'optimalité}

Le programme du planificateur consiste à déterminer les trajectoires de consommations sectorielles de chaque type d'énergie et les trajectoires des flux de captures à la source et dans l'atmosphère qui maximisent la fonction de bien-être social $W$ sous l'ensemble des contraintes (1)-(7).

On note $\lambda_{X}$ la variable d'état adjointe à la variable $X$ (i.e. la rente de rareté du charbon), $\lambda_{Z}$ la variable d'état adjointe à $Z$ en valeur absolue (i.e. le coût marginal social de la pollution), $\gamma$ les multiplicateurs de Lagrange associés aux contraintes d'inégalités sur les variables de commande et $v$ le multiplicateur de Lagrange associé à la contrainte de plafond sur le stock de pollution. Comme il est d'usage dans ce type de modèles, la contrainte de non-négativité sur la variable $X$ n'est pas explicitement prise en compte. Le Lagrangien du programme s'écrit donc: 


$$
\begin{gathered}
L=\sum_{i} S_{i}\left(x_{i}, y_{i}\right)-c_{e} s_{e}-c_{a} s_{a}-\lambda_{X}\left(x_{1}+x_{2}\right)-\lambda_{Z}\left[\zeta\left(x_{1}+x_{2}\right)-\left(s_{e}+s_{a}\right)-\alpha Z\right] \\
+v(\bar{Z}-Z)+\sum_{i} \gamma_{x_{i}} x_{i}+\sum_{i} \gamma_{y_{i}} y_{i}+\gamma_{s_{e}} s_{e}+\bar{\gamma}_{s_{e}}\left(\zeta x_{1}-s_{e}\right)+\gamma_{s_{a}} s_{a}
\end{gathered}
$$

Les conditions du premier ordre sont :

$$
\begin{gathered}
u^{\prime}\left(x_{1}+y_{1}\right)=c_{x}+\lambda_{X}+\zeta\left(\lambda_{z}-\bar{\gamma}_{s_{e}}\right)-\gamma_{x_{1}} \\
u^{\prime}\left(x_{2}+y_{2}\right)=c_{x}+\lambda_{X}+\zeta \lambda_{z}-\gamma_{x_{2}} \\
u^{\prime}\left(x_{i}+y_{i}\right)=c_{y}-\gamma_{y_{i}}, \quad i=1,2 \\
c_{e}=\lambda_{z}-\bar{\gamma}_{s_{e}}+\gamma_{s_{e}} \\
c_{a}=\lambda_{z}+\gamma_{s_{a}} \\
\dot{\lambda}_{X}=\rho \lambda_{X} \\
\dot{\lambda}_{z}=(\rho+\alpha) \lambda_{z}-v
\end{gathered}
$$

auxquelles il faut ajouter les conditions de Kuhn et Tucker associées aux différentes contraintes d'inégalité ainsi que les conditions de transversalité suivantes :

$$
\begin{aligned}
& \lim _{t \uparrow \infty} e^{-\rho t} \lambda_{X}(t) X(t)=0 \\
& \lim _{t \uparrow \infty} e^{-\rho t} \lambda_{Z}(t) Z(t)=0
\end{aligned}
$$

Analysons à présent ces conditions, en commençant par les équations (13) et (14) qui établissent respectivement la dynamique optimale de la rente de rareté du charbon et de la valeur marginale de la pollution.

Rente de rareté du charbon :

Comme dans tout modèle " à la Hotelling " avec coût marginal d'extraction constant, la valeur marginale du charbon en terre, i.e. sa rente de rareté $\lambda_{X}$, doit croître au rythme du taux d'actualisation $\rho$ d'après (13). Sa trajectoire est donc donnée par $\lambda_{X}(t)=\lambda_{X_{0}} e^{\rho t}$, où $\lambda_{X_{0}} \equiv \lambda_{X}(0)$. De plus, si la valeur initiale du charbon en terre est strictement positive, $\lambda_{X_{0}}>0$, la condition (15) implique que les réserves doivent être intégralement épuisées le long du sentier optimal d'exploitation.

Valeur marginale des émissions de carbone :

Puisque le niveau initial de concentration atmosphérique en carbone est supposé inférieur au plafond prescrit et en posant que les réserves initiales de charbon sont suffisamment importantes de sorte que la régénération naturelle ne puisse compenser à 
court terme le flux d'émissions polluantes, alors le stock atmosphériques de carbone augmente à mesure qu'est utilisé du charbon dans l'un ou l'autre des deux secteurs (avec des rythmes d'accroissement plus ou moins élevés selon que sont déployées ou non les technologies d'abattement). La trajectoire de $Z$ admet donc trois grandes phases. La première phase est une phase de montée progressive au plafond ; la seconde est une phase dite " au plafond " au cours de laquelle $Z$ est constant et égal à $\bar{Z}$; la dernière phase est caractérisée par une diminution des émissions de carbone du fait de l'épuisement progressif des réserves de charbon et donc le stock $Z$ retombe en-deçà du plafond de concentration autorisé. On suppose que les réserves initiales de charbon sont suffisamment importantes et/ou que le niveau du plafond est suffisamment bas pour qu'apparaissent successivement ces trois phases. Les répercussions sur la dynamique de la valeur marginale sociale de la pollution sont les suivantes (cf. Chakravorty et al., 2006) :

- Au cours de la phase de montée au plafond, la condition (14) stipule que $\lambda_{Z}$ croit au taux d'actualisation " modifié " $\rho+\alpha$ (afin de prendre en compte le fait que toute unité émise dans l'atmosphère est assimilée au taux $\alpha): \lambda_{Z}(t)=\lambda_{Z_{0}} e^{(\rho+\alpha) t}$, avec $\lambda_{Z_{0}} \equiv \lambda_{Z}(0)$.

- Durant la phase au plafond, le stock de pollution étant stabilisé au niveau $\bar{Z}$, le taux de croissance de $\lambda_{Z}$ doit être inférieur à $\rho+\alpha$. Comme nous le verrons dans la section suivante, $\lambda_{Z}$ doit en fait décroître par paliers.

- Enfin, lorsque le plafond de concentration est définitivement quitté, la pollution ne représente plus aucune contrainte pour la société et sa valeur marginale est nulle ${ }^{13}$ : $\lambda_{Z}=0$.

\section{Compétitivité relative des énergies primaires :}

Les conditions (8)-(10) égalisent le surplus marginal brut que retire chaque secteur de la consommation de l'une ou l'autre des deux sources d'énergie primaire à son coût marginal complet d'utilisation. Dans le cas du charbon, ce coût marginal inclut le coût d'approvisionnement $c_{x}$, la rente de rareté $\lambda_{X}$, et le coût marginal social de la pollution. Les deux premiers termes sont communs aux deux secteurs. En revanche, le poids de la contrainte de plafond sera perçu différemment par les deux secteurs en fonction de leurs niveaux respectifs d'abattement. Tant qu'aucune opération d'abattement n'est entreprise, le coût marginal complet d'utilisation du charbon sera le même dans chaque secteur. Dans le cas contraire, le prix de l'énergie au consommateur peut être différent selon les secteurs. Lorsque les deux secteurs consomment le flux d'énergie solaire, leurs surplus marginaux sont identiques et égaux au coût marginal d'approvisionnement $c_{y}$ (cf. équation (10)).

\footnotetext{
${ }^{13}$ Ce résultat se démontre facilement en utilisant la condition de transversalité (16).
} 
Dans chaque secteur, le choix de consommation entre ces deux sources primaires dépend de leur compétitivité relative respective et résulte donc de la comparaison de leurs coûts marginaux complets d'utilisation. Le coût d'utilisation de l'énergie solaire est constant et celui du charbon est non-décroissant du fait des différents termes qui le composent. Une utilisation simultanée de ces deux sources énergétiques n'est donc possible que lors de phases au plafond, lorsque la décroissance de la valeur marginale de la pollution compense la croissance de la rente de rareté du charbon. Néanmoins, afin de centrer l'analyse sur la réduction des émissions de carbone par capture, et non par substitution énergétique, on suppose que le coût d'utilisation du substitut solaire est suffisamment élevé pour que la trajectoire optimale de consommation d'énergie de chaque secteur soit séparée en deux grandes périodes : une première période durant laquelle seul le charbon est utilisé, suivie d'une période d'utilisation exclusive du flux d'énergie solaire. Les scenarii pour lesquels une utilisation simultanée des deux sources d'énergie est possible, au moins dans un secteur, sont décrits dans Amigues et al. (2012-b).

Options de capture du carbone :

Les conditions (11) et (12) établissent la relation entre la valeur marginale de la pollution et les coûts marginaux de capture, que ce soit à la source des émissions pour le secteur 1 ou dans l'atmosphère pour le secteur 2 . Tant que la contrainte de plafond est effective, i.e. tant que le stock de pollution atmosphérique $Z$ n'est pas retombé en dessous de $\bar{Z}$, chaque secteur subit le poids de la contrainte par l'imposition d'un coût additionnel d'utilisation du charbon égal à $\lambda_{z}$. Dans le cas du secteur 1 , ce poids peut-être atténué par la capture de tout ou partie des émissions. Le secteur 2 ne peut traiter lui-même ses rejets, mais la société peut décider de déployer la capture atmosphérique lorsque cette option devient compétitive. Clairement, la compétitivité de l'une ou l'autre de ces deux options d'abattement dépend de la comparaison entre le coût marginal de capture associé et le coût social de la pollution. Tant que le coût de capture est supérieur au coût de la pollution, il est socialement préférable de subir le poids de la contrainte environnementale plutôt que d'abattre. En revanche, il sera optimal de déployer la technologie en question dans le cas contraire. Selon la valeur maximale $\bar{\lambda}_{z} \equiv \operatorname{argmax}_{t} \lambda_{Z}(t)$ du coût social de la pollution, ce cadre d'analyse coût-efficacité implique donc trois types de scenarii :

i) $\bar{\lambda}_{Z}<c_{e}<c_{a}$ : aucune opération de capture n'est engagée le long du sentier optimal, que ce soit à la source des émissions pour le secteur 1 ou dans l'atmosphère pour le secteur 2 .

ii) $\quad c_{e} \leq \bar{\lambda}_{Z}<c_{a}$ : il est optimal de capturer les émissions du secteur 1 par CSC (avec capture totale si $\left.c_{e}<\lambda_{Z}(t)\right)$, mais pas de pratiquer la capture atmosphérique.

iii) $\quad c_{e}<c_{a} \leq \bar{\lambda}_{Z}$ : il peut être optimal de déployer les deux modes de capture. De plus, la totalité des émissions du secteur 1 doit être séquestrée puisque $c_{e}<\bar{\lambda}_{Z}$. 
Dans la section suivante, nous restreignons l'analyse au scenario le plus pertinent, c'est-à-dire au sentier optimal le long duquel il est optimal de déployer les deux formes de capture. Une étude des autres scenarios possibles est menée dans Amigues et al. (2012-b).

\section{Scenario optimal avec mobilisation des deux technologies de capture}

Le sentier optimal présente une succession de 8 phases, chacune étant caractérisée par le fait que la contrainte de plafond est saturée ou non, que les utilisateurs consomment du charbon ou de l'énergie solaire et enfin, que chacune des deux technologies de capture est mobilisée ou non. Les détails techniques incluant les expressions des trajectoires optimales de l'ensemble des variables de l'économie (prix de l'énergie, quantités consommées et flux d'abattement), les conditions d'existence de la solution optimale et la caractérisation des composantes endogènes du modèle (valeurs initiales des multiplicateurs et dates de transition entre deux phases successives) font l'objet d'un appendice mathématique séparé. Celui-ci peut être communiqué sur demande.

Phase 1 : Avant plafond et sans abattement

Au cours de cette phase, le coût marginal de la pollution est tel que $\lambda_{Z}(t)=$ $\lambda_{z_{0}} e^{(\rho+\alpha) t}<c_{e}<c_{a}$. Aucun contrôle des émissions n'est alors entrepris car il serait plus coûteux d'abattre que de supporter le coût marginal social de la pollution. Le prix de l'énergie au consommateur est le même dans chaque secteur. II se compose du coût marginal d'approvisionnement en charbon, de la rente de rareté et du coût marginal social de la pollution : $p_{i}(t)=p(t)=c_{x}+\lambda_{X_{0}} e^{\rho t}+\zeta \lambda_{z_{0}} e^{(\rho+\alpha) t}, i=1,2$. Ce prix est croissant au cours du temps. Les deux secteurs ayant la même fonction de demande, ils consomment chacun la même quantité de charbon $x_{i}(t)=x(t)=q^{d}(p(t))$. Les émissions totale de carbone sont égales à $2 \zeta x(t)$ et impliquent un accroissement du stock de pollution.

La trajectoire de $\lambda_{Z}$ étant croissante, cette première phase s'achève lorsque le coût marginal de la pollution atteint le niveau $c_{e}$, ce qui déclenche l'utilisation du CSC dans le secteur 1 . Notons qu'à cet instant, le plafond de concentration atmosphérique de carbone n'est pas encore atteint.

Phase 2 : Avant plafond et séquestration de la totalité des émissions du secteur 1

Le coût marginal social de la pollution étant toujours croissant puisque le plafond n'est pas encore atteint, il devient à présent supérieur au coût marginal de CSC pour le secteur 1, mais demeure strictement inférieur au coût marginal de la capture atmosphérique : $c_{e}<\lambda_{Z_{0}} e^{(\rho+\alpha) t}<c_{a}$. Il est alors moins coûteux pour le secteur 1 d'abattre ses émissions que de subir la charge de la contrainte environnementale. Son surplus 
marginal brut s'élève donc à $p_{1}(t)=c_{x}+\lambda_{X_{0}} e^{\rho t}+\zeta c_{e}$. II consomme $x_{1}(t)=q^{d}\left(p_{1}(t)\right)$ unités de charbon et séquestre la totalité des émissions générées : $s_{e}(t)=\zeta x_{1}(t)$.

Il est impossible pour le secteur 2 de capturer ses émissions directement à la source. Il supporte donc seul le coût environnemental et continue de percevoir un surplus marginal brut correspondant à $p_{2}(t)=c_{x}+\lambda_{X_{0}} e^{\rho t}+\zeta \lambda_{z_{0}} e^{(\rho+\alpha) t}$. Puisque durant cette phase $p_{2}(t)>p_{1}(t)$, la consommation de charbon du secteur $2, x_{2}(t)=q^{d}\left(p_{2}(t)\right)$, est inférieure à celle du secteur 1 .

La quantité totale de carbone émise dans l'économie correspond aux seules émissions du secteur 2. Le stock atmosphérique de carbone $Z(t)$ continue de croître et son coût social $\lambda_{Z}(t)$, d'augmenter. La phase se termine lorsque ce stock atteint le plafond de concentration autorisé et, simultanément, le coût marginal social de la pollution atteint le niveau du coût marginal de la capture atmosphérique.

Phase 3 : Au plafond, avec mobilisation des deux technologies de capture

Cette phase est caractérisée par l'entrée en action de la capture atmosphérique, rendue compétitive puisque $\lambda_{Z}(t)=c_{a}$ et par la stabilisation du stock de pollution atmosphérique à son niveau maximal autorisé $\bar{Z}$. Durant cette phase, les deux technologies de capture sont donc mobilisées.

Puisque $\lambda_{Z}(t)=c_{a}>c_{e}$, le secteur 1 continue d'abattre la totalité de ses émissions: $s_{e}(t)=\zeta x_{1}(t)$. Son surplus marginal est donc toujours $p_{1}(t)=c_{x}+\lambda_{X_{0}} e^{\rho t}+\zeta c_{e}$ et sa consommation de charbon, $x_{1}(t)=q^{d}\left(p_{1}(t)\right)$.

A présent, les émissions du secteur 2 sont directement capturées dans l'atmosphère. Celui-ci fait face à un prix de l'énergie au consommateur $p_{2}(t)=c_{x}+\lambda_{X_{0}} e^{\rho t}+\zeta c_{a}$ supérieur à $p_{1}(t)$ et consomme donc une quantité de charbon $x_{2}(t)=q^{d}\left(p_{2}(t)\right)$ inférieure à celle du secteur 1 . Puisque les émissions résiduelles du secteur 1 sont nulles, la capture atmosphérique ne va concerner que cette partie des émissions du secteur 2 qui permet la stabilisation du stock de pollution au niveau $\bar{Z}: s_{a}(t)=\zeta x_{2}(t)-\alpha \bar{Z}<\zeta x_{2}(t)$, c'est-à-dire les émissions résiduelles non absorbées par le milieu naturel. De plus, les émissions du secteur 2 diminuant puisque $p_{2}(t)$ augmente, la quantité de carbone $s_{a}(t)$ ainsi capturée est décroissante.

La phase s'achève lorsque $s_{a}(t)=0$. La capture atmosphérique cesse alors d'être profitable et le coût marginal social de la pollution devient inférieur à $c_{a}$, impliquant une restriction de la consommation de charbon pour le secteur 2 égale à $x_{2}(t)=\alpha \bar{Z} / \zeta \equiv \bar{x}$.

Phase 4 : Au plafond, avec séquestration de la totalité des émissions du secteur 1

Au cours de cette phase, le coût marginal social de la pollution est tel que $c_{e}<$ $\lambda_{Z}(t)<c_{a}$. La capture atmosphérique n'est donc plus une option compétitive. En revanche, il est toujours optimal de capturer la totalité des émissions du secteur 1 . Le surplus marginal 
brut, la consommation de charbon et l'abattement de ce secteur suivent donc les mêmes trajectoires qu'au cours de la phase précédente.

Le secteur 2 subit seul le coût de la contrainte environnementale. De plus, puisque le stock de pollution est maintenu à son plafond maximal autorisé, sa consommation de charbon est "bloquée " au niveau $\bar{x}$. Le surplus marginal brut que retire le secteur 2 de cette consommation est $p_{2}(t)=c_{x}+\lambda_{X_{0}} e^{\rho t}+\zeta \lambda_{z}(t)=u^{\prime}(\bar{x})$. Puisque $c_{e}<\lambda_{z}(t)$, on a $p_{1}(t)<p_{2}(t)$ et donc $x_{1}(t)>x_{2}(t)$. Notons aussi que, puisque $p_{2}(t)$ est constant au cours de la phase et que $\lambda_{X_{0}} e^{\rho t}$ est croissant, le coût marginal social du carbone doit décroître. La phase s'achève lorsque celui-ci atteint le niveau $c_{e}$.

\section{Phase 5 : Au plafond, avec séquestration partielle des émissions du secteur 1}

Cette phase, au cours de laquelle $\lambda_{Z}(t)=c_{e}$, est marquée par l'arrêt progressif du CSC dans le secteur 1 . Les deux secteurs ont à présent le même surplus marginal brut: $p_{i}(t)=p(t)=c_{x}+\lambda_{X_{0}} e^{\rho t}+\zeta c_{e}>u^{\prime}(\bar{x})$. Par conséquent, ils utilisent la même quantité de charbon $x(t)=q^{d}(p(t))$ comprise entre $\bar{x} / 2$ et $\bar{x}$. Les émissions totales s'élèvent à $2 \zeta x(t)$ et, puisque le stock de pollution est toujours bloqué au plafond, l'abattement des émissions du secteur 1 correspond à $s_{e}(t)=\zeta[2 x(t)-\bar{x}]<\zeta x(t)$. Le secteur 1 ne capture donc plus qu'une partie de ses rejets. De plus, ce niveau d'abattement décroît au cours du temps. La phase s'achève avec l'arrêt définitif du CSC lorsque $s_{e}(t)=0$. A cet instant, le coût marginal de la pollution recommence à décroître et repasse en dessous de $c_{e}$.

\section{Phase 6 : Au plafond sans aucun abattement}

Puisque capturer ses émissions est devenu trop coûteux pour le secteur 1 , plus aucune opération d'abattement n'est menée. La contrainte de plafond étant toujours saturée, la consommation totale de charbon est " bloquée " à $\bar{x}$. Les deux secteurs ayant la même fonction de demande, chacun utilise la même quantité $\bar{x} / 2$. Le surplus marginal brut retiré par chaque secteur est constant et s'élève à $p_{i}(t)=u^{\prime}(\bar{x} / 2)=c_{x}+\lambda_{X_{0}} e^{\rho t}+\zeta \lambda_{Z}(t)$. Le poids de la contrainte environnementale est à présent supporté équitablement par les deux secteurs. Ce coût diminue au cours du temps. La phase se termine lorsque celui-ci devient nul et, simultanément, lorsque le stock de pollution quitte définitivement le seuil maximal de concentration $\bar{Z}$.

\section{Phase 7 dite phase « à la Hotelling»}

A partir de cette phase, la concentration atmosphérique de carbone n'est plus contraignante pour la société et plus aucun coût additionnel ne doit être imputé pour l'émission de rejets carbonés. La demande en énergie de chaque secteur est toujours exclusivement fournie par le charbon. On retrouve donc un principe de fonctionnement de l'économie "à la Hotelling ". Le surplus marginal brut, identique aux deux secteurs, est 
$p_{i}(t)=p(t)=c_{x}+\lambda_{X_{0}} e^{\rho t}$ (le surplus marginal net croît au rythme du taux d'actualisation) et I'utilisation de charbon associée s'élève à $x_{i}(t)=q^{d}(p(t))$. Cette trajectoire de consommation décroît progressivement et la phase se termine lorsqu'elle devient nulle. A cet instant, les réserves de charbon doivent être entièrement épuisées et, simultanément, le prix de l'énergie doit avoir atteint $c_{y}$, niveau à partir duquel l'énergie solaire devient compétitive, afin de garantir la continuité du sentier de consommation.

\section{Phase 8 : Régime permanent d'utilisation de l'énergie solaire}

Au cours de cette dernière phase, l'économie consomme indéfiniment le flux d'énergie solaire. Le surplus sectoriel est alors constant et égal à $c_{y}$. La consommation sectorielle d'énergie est également constante : $y_{i}(t)=\tilde{y}$. Puisque plus aucun rejet de carbone n'est émis, le stock atmosphérique décroît par processus d'auto-régénération, et tend asymptotiquement vers 0 .

Ces différentes phases sont illustrées par la figure 2 .

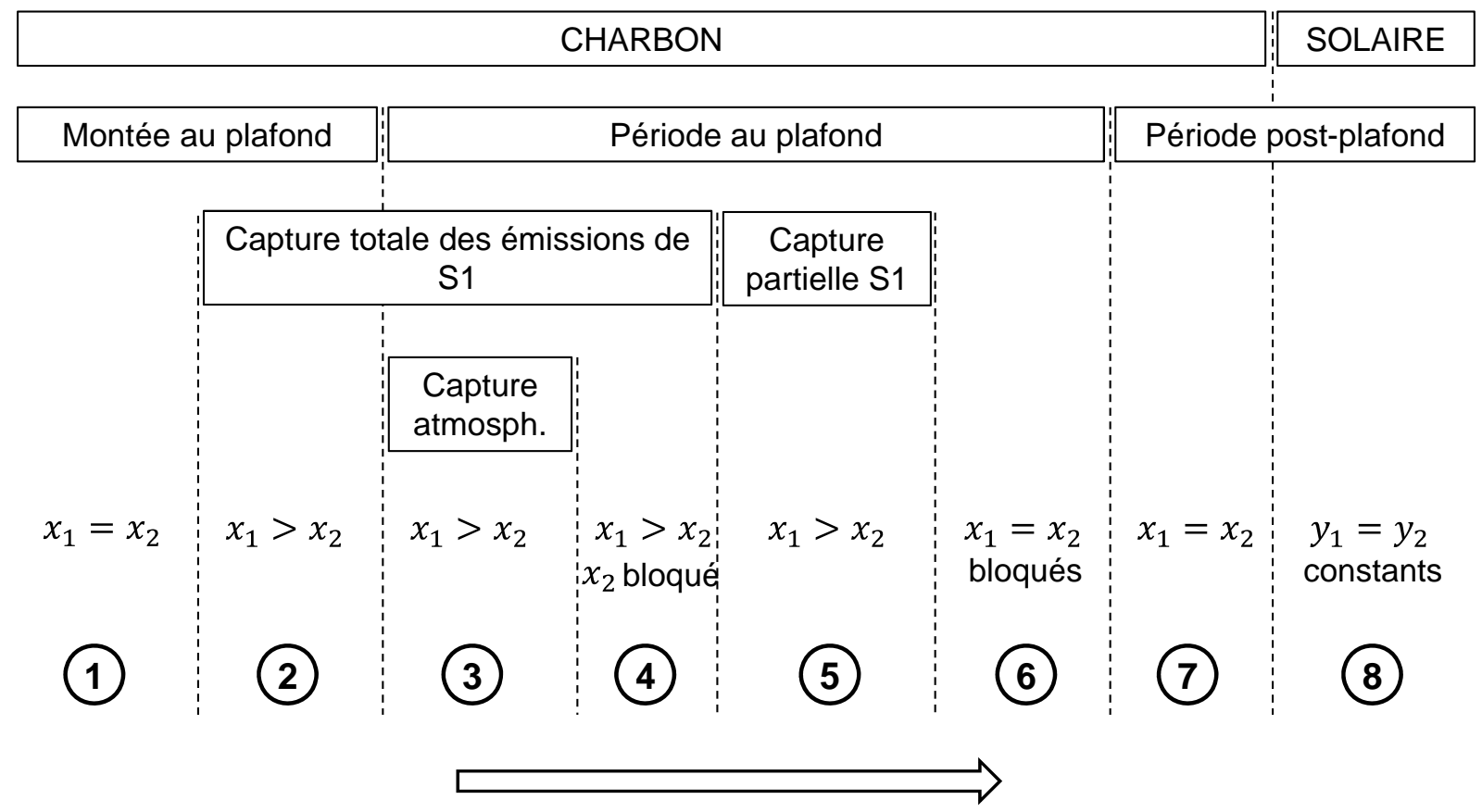

Figure 2 : Résumé des différentes phases du sentier optimal

Les principaux enseignements de ce type de scenario optimal sont résumés dans la proposition suivante. 
Proposition 1 : Lorsque le sentier optimal suivi par l'économie nécessite la mobilisation des deux technologies de capture, alors :

i) La capture des émissions du secteur 1 doit débuter avant que le plafond de concentration atmosphérique de carbone ne soit atteint.

ii) Il peut être optimal pour le secteur 1 d'abattre la totalité de ses émissions, au moins au début.

iii) La capture atmosphérique ne doit être mobilisée qu'au moment où le plafond est atteint, et pour une durée plus courte que celle du CSC.

iv) Les surplus marginaux sectoriels sont différents au cours des phases où au moins l'un des deux secteurs a recours à l'abattement.

Ces résultats contrastent avec ceux obtenus par Chakravorty et al. (2006) et Lafforgue et al. (2008-a). En effet, en utilisant un cadre d'analyse similaire mais en supposant l'existence d'un seul type de consommateur, ces deux modèles montrent qu'il n'est jamais optimal de déployer la technologie d'abattement, quelle qu'elle soit, avant d'avoir atteint le seuil critique de pollution, ni d'abattre la totalité du flux d'émissions.

\section{Taxe carbone optimale et poids du changement climatique pour chaque secteur}

Nous discutons à présent du profil temporel du coût marginal social de la pollution, c'est-à-dire de la taxe sur les rejets de $\mathrm{CO}_{2}$ nets qu'il faudrait implémenter dans une économie décentralisée et purement concurrentielle pour induire les trajectoires optimales d'émissions. Nous analysons ensuite le coût total du changement climatique subi par chaque secteur, qui inclut la taxe carbone et le coût d'abattement des émissions polluantes.

\subsection{Profil temporel de la taxe carbone optimale}

Le profil temporel du coût marginal social de la pollution correspondant au scenario optimal décrit dans la Section 4 est illustré par la Figure 3. 


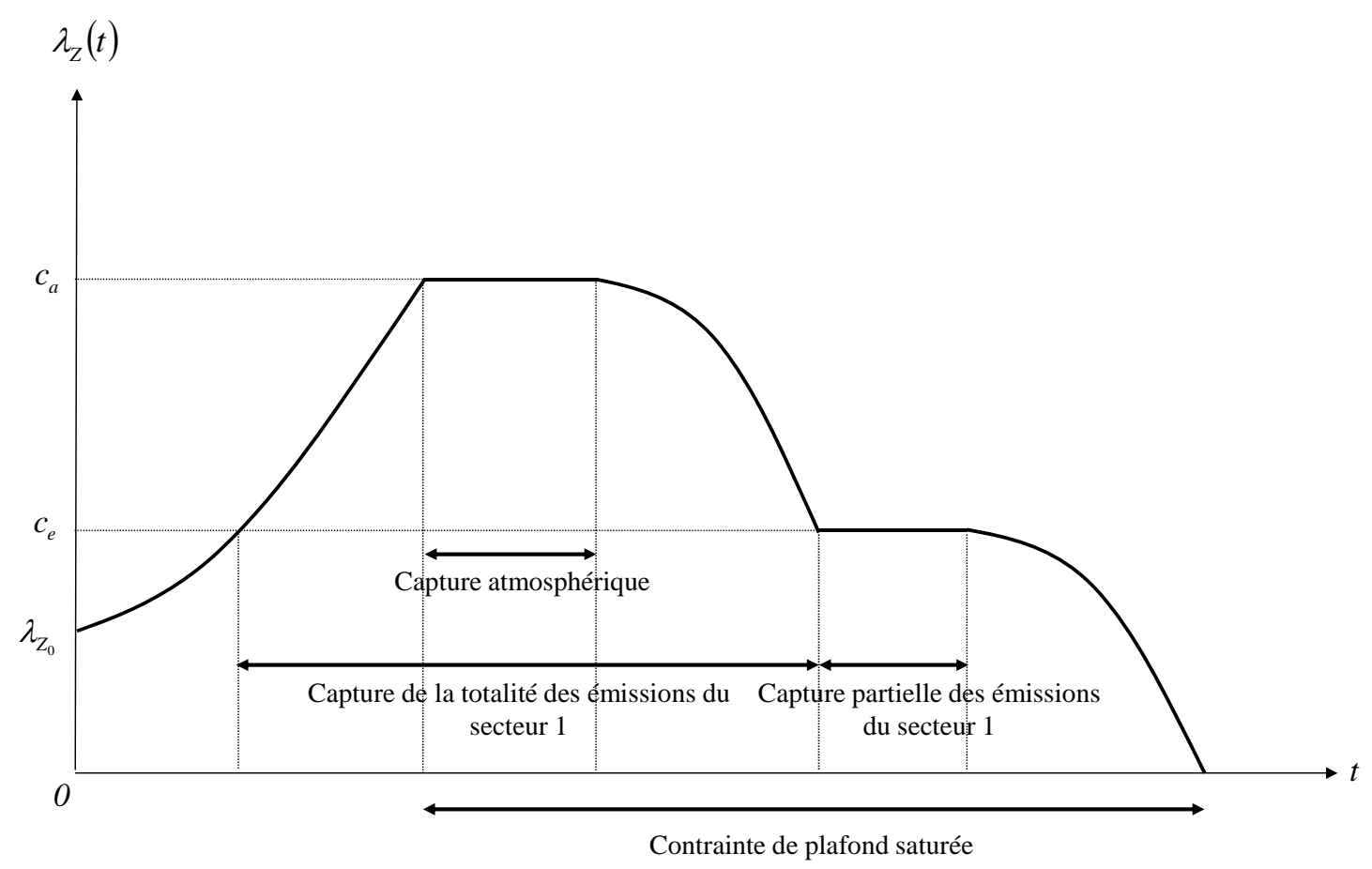

Figure 3 : Profil temporel de la taxe carbone optimale

La taxe unitaire est tout d'abord croissante durant les phases 1 et 2, mais bornée supérieurement par le coût marginal d'abattement le plus élevé des deux technologies disponibles, c'est-à-dire $c_{a}$. Ce niveau de taxe est atteint lorsque il devient optimal d'avoir recours à la capture atmosphérique et, simultanément, lorsque le plafond de concentration atmosphérique de pollution est atteint. Tant que l'externalité environnementale est contraignante pour l'économie, étant donné que chaque secteur a en permanence le choix entre abattre ses émissions ou les laisser se diffuser dans l'atmosphère et subir le coût social associé, le taux maximal de la taxe carbone qu'il convient de fixer est nécessairement déterminé par le coût marginal d'abattement le plus élevé.

Au cours des différentes phases au plafond, la taxe carbone est soit constante, soit décroissante. Premièrement, durant la phase 3 , tant que les émissions du secteur 2 sont réduites par capture atmosphérique, un niveau de taxe fixé à $c_{a}$ est suffisant pour induire une capture optimale dans ce secteur étant donné que, simultanément, le secteur 1 séquestre la totalité de ses rejets polluants. La même conclusion s'applique à la phase 5 pour le secteur 1, la taxe étant alors fixé à $c_{e}$ étant donné que le secteur 2 n'abat plus ses émissions. Entre ces deux phases et au cours de la dernière phase au plafond respectivement au cours des phases 4 et 6 - le taux de taxe décroît. Ceci est dû tout d'abord à l'effet d'Hotelling qui implique un prix du charbon croissant à mesure que les réserves diminuent, et ensuite au fait que la consommation totale de charbon est bloquée au cours de ces phases puisque la contrainte de plafond est toujours saturée.

Enfin, une fois que le stock de pollution a commencé à diminuer et s'éloigne progressivement de son seuil critique, les émissions de carbone $n^{\prime}$ induisent plus aucune externalité et une taxe visant à les contrôler n'est plus nécessaire. 


\subsection{Coût sectoriel total induit par le changement climatique}

Supposons que la taxe carbone décrite plus haut soit implémentée. La charge fiscale qu'elle représente pour chaque secteur, notée $\Gamma_{i}$, correspond au volume de ses émissions nettes multiplié par le taux de taxe en question. On obtient donc: $\Gamma_{1}(t)=\left[\zeta x_{1}(t)-\right.$ $\left.s_{e}(t)\right] \lambda_{Z}(t)$ et $\Gamma_{2}(t)=\left[\zeta x_{2}(t)-s_{a}(t)\right] \lambda_{Z}(t)$. Par ailleurs, pour réduire ou se soustraire à cette charge, les deux secteurs ont la possibilité de contrôler leurs émissions. Le coût total de capture, noté $S_{i}$, s'élève donc à $S_{1}(t)=s_{e}(t) c_{e}$ pour le secteur 1 et à $S_{2}(t)=s_{a}(t) c_{a}$ pour le secteur 2. Par conséquent, le coût total induit par l'externalité environnementale est défini pour chaque secteur $i$ par $B_{i}(t) \equiv \Gamma_{i}(t)+S_{i}(t)$. La composition de cette charge totale pour chaque secteur et pour chaque phase du scenario optimal est détaillée dans le Tableau 1 ci-dessous. La Figure 4 illustre leur profil temporel.

\begin{tabular}{c|c|c} 
Phases & Secteur 1 & Secteur 2 \\
\hline 1 & $B_{1}=\Gamma_{1}$ & $B_{2}=\Gamma_{2}=B_{1}$ \\
2 & $B_{1}=S_{1}$ & $B_{2}=\Gamma_{2}>B_{1}$ \\
3 & $B_{1}=S_{1}$ & $B_{2}=\Gamma_{2}+B_{2}>B_{1}$ \\
4 & $B_{1}=S_{1}$ & $B_{2}=\Gamma_{2}>B_{1}$ \\
5 & $B_{1}=\Gamma_{1}+S_{1}$ & $B_{2}=\Gamma_{2}=B_{1}$ \\
6 & $B_{1}=\Gamma_{1}$ & $B_{2}=\Gamma_{2}=B_{1}$ \\
7 et 8 & $B_{1}=0$ & $B_{2}=0$
\end{tabular}

Tableau 1 : Décomposition du coût total du carbone par secteur 


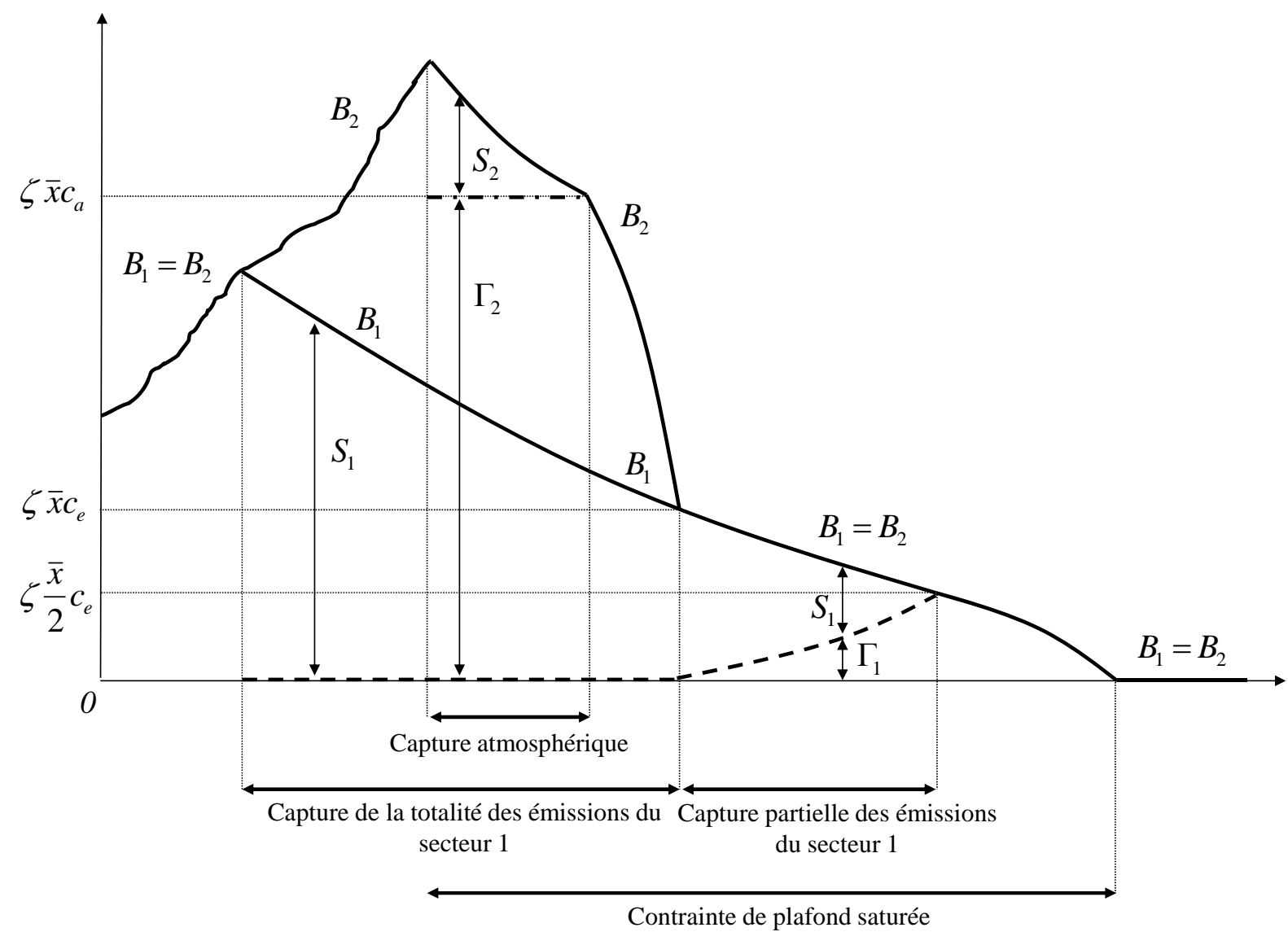

Figure 4 : Coût total du carbone pour chaque secteur

L'allure générale des $B_{i}$ est la suivante. Au cours des phases précédents le plafond (phases 1 et 2 ), leurs trajectoires peuvent être non-monotones selon les propriétés de la fonction de demande, en particulier son élasticité (non spécifiée dans ce modèle). Une fois le plafond atteint, le coût total du carbone est, sans ambiguïté, décroissant. Enfin, il devient nul une fois le plafond définitivement quitté.

Quel que soit le secteur considéré, le coût total de l'externalité correspond au seul paiement de la taxe tant qu'aucun dispositif d'abattement n'est mis en place. Lors des phases de capture de la totalité de ses rejets (phases 2, 3 et 4), le secteur 1 s'affranchit entièrement $\mathrm{du}$ paiement de la taxe carbone et doit donc supporter un fardeau correspondant à ses seuls coûts de séquestration. Au cours de ces trois phases, puisque les émissions du secteur 1 sont décroissantes et que le coût marginal du CSC est constant, le coût total du carbone est décroissant pour ce secteur. Au cours de la phase suivante (phase 5), il n'est plus optimal pour le secteur 1 de séquestrer la totalité de ses émissions et la charge totale qu'il subit est composée du coût de capture et du paiement de la taxe sur les émissions non capturées. Ses émissions brutes diminuent et son flux de séquestration décroît également mais à un rythme plus soutenu ce qui entraîne une hausse de ses émissions nettes. Par conséquent, le coût de séquestration du secteur 1 diminue et la charge fiscale qu'il subit en s'acquittant de la taxe sur ses émissions résiduelles augmente, mais à un 
rythme moins élevé. L'effet combiné de ces deux évolutions se traduit par une baisse de la charge totale supportée par le secteur 1 au cours de cette phase. Lors de la dernière phase au plafond (phase 6), le secteur 1 ayant cessé toute capture des rejets, cette charge correspond au seul paiement de la taxe.

Durant la phase de capture atmosphérique (phase 3), une partie seulement des émissions du secteur 2 est séquestrée. La charge totale que supporte ce secteur comprend alors le coût de capture et le paiement de la taxe sur les émissions non séquestrées. Cette charge est constante au cours de la phase en question car i) le taux de taxe est constant et égal à $c_{a}$ et ii) les émissions résiduelles du secteur 2 sont "bloquées " au niveau $\bar{x}$ puisque le secteur 1 capture la totalité de ses rejets. Une fois cette phase de capture achevée et jusqu'à la fin de la période au plafond (phases 4,5 et 6 ), le secteur 2 supporte uniquement le poids de la taxe carbone.

Deux points importants méritent d'être souligné. Premièrement, les recettes fiscales totales provenant de la taxe carbone, i.e. $\Gamma_{1}(t)+\Gamma_{2}(t)$, ont une trajectoire décroissante et discontinue. En effet, celle-ci fait un saut vers le bas à chaque fois qu'un secteur commence à abattre ses émissions. Ce résultat est dû à l'hypothèse de coûts de capture constants mais, de façon générale, toute politique environnementale visant à pénaliser les rejets polluants devrait prendre en considération la possibilité qu'ont les pollueurs d'entreprendre des opérations d'abattement pour se soustraire à ladite politique. Deuxièmement, puisque le secteur 2 n'a accès qu'à la technologie de capture la plus onéreuse, sa contribution fiscale et la charge totale qu'il supporte sont supérieures ou égales à celles du secteur 1 alors même que la demande d'énergie est la même pour ces deux secteurs.

\section{Conclusion}

Dans un modèle de gestion de ressources " à la Hotelling ", nous avons déterminé les politiques optimales de capture des émissions carbonées de deux secteurs se différenciant par leur technologie d'abattement. Le secteur 1 (industries lourdes ou centrales électriques thermiques par exemple), dont les émissions sont suffisamment concentrées pour être traitées à la source, a accès au CSC mais pas le secteur 2 (transport par exemple) dont les émissions sont plus diffuses. La seule possibilité de réduire la pollution de ce secteur consiste à capturer le carbone dans l'atmosphère, solution qui s'avère plus coûteuse que le CSC. Enfin, les deux secteurs font face à une contrainte globale de limitation de la concentration atmosphérique en carbone.

Dans ce cadre d'analyse, en supposant tous les coûts constants, nous avons identifié un scenario optimal dans lequel le secteur 1 commence à capturer ses émissions avant l'atteinte du plafond de concentration autorisé et abat la totalité de ses rejets durant une première période. Ce résultat est contraire aux conclusions de Chakravorty et al. (2006) et 
de Lafforgue et al. (2008-a) selon lesquelles, en présence d'un seul secteur d'activité, tout abattement ne doit commencer qu'une fois le plafond atteint et ne doit porter que sur une partie seulement des rejets polluants.

Cette différence est une conséquence directe de l'hypothèse d'hétérogénéité des utilisateurs d'énergie, la technologie de capture la moins coûteuse étant accessible à certains secteurs d'activité tels que les centrales électriques thermiques, les aciéries ou les cimenteries, mais pas à d'autres, comme le secteur des transports. Cette hypothèse contraint le potentiel de capture du CSC à être au plus égal aux seuls rejets du secteur 1 et, par conséquent, à être en permanence inférieur aux émissions totales générées par l'économie. Lorsque les coûts marginaux d'abattement sont constants, la seule politique optimale pour l'économie consiste à séquestrer la totalité des émissions du secteur 1 et à utiliser, en complément et de façon transitoire, la capture atmosphérique pour alléger le poids de la contrainte de plafond lorsque celle-ci devient saturée. En outre, que la capture atmosphérique soit déployée ou non, il est toujours optimal de démarrer le CSC avant l'atteinte du plafond, et ce pour les mêmes raisons que celles invoquées plus haut. Notons qu'il n'est optimal de déployer la capture atmosphérique qu'une fois le plafond atteint, résultat qui concorde à présent avec celui de Chakravorty et al. (2006).

Du fait des différentes phases de capture dans chaque secteur, le profil temporel de la taxe carbone optimal apparaît plus complexe que dans les modèles standards «à plafond ". Le taux de taxe doit tout d'abord croître au cours du temps tant que le plafond n'est pas atteint. II décroît ensuite par pallier lors des phases au plafond, chaque pallier correspondant à une phase d'abattement partiel selon chacune des deux technologies. II est également important de rappeler que cette taxe doit être unique, i.e. que le même profil de taxe doit s'appliquer à tous les secteurs quel qu'en soient le nombre et la nature, même si le prix au consommateur diffère d'un secteur à l'autre. Par ailleurs, l'instauration d'une valeur carbone suffisamment élevée (taxe ou prix dans un marché de droits) est une condition nécessaire pour garantir une compétitivité minimale de la séquestration et en assurer ainsi le développement. Cela se traduit dans notre modèle par un niveau de plafond suffisamment contraignant pour qu'il soit optimal de mobiliser le CSC et la capture atmosphérique. A l'aide d'un modèle d'évaluation intégrée, Grimaud et al. (2011) montrent que préconiser un seuil de concentration atmosphérique de carbone supérieur à 550ppmv serait insuffisant pour inciter au développement de la séquestration.

Notons enfin que le secteur 1 échappe au paiement de la taxe tant qu'il capture la totalité de ses émissions, cette charge étant transférée au secteur 2. Cette divergence entre secteurs s'explique par le fait que le secteur 2 bénéficie d'une sorte "d'effet externe positif » du fait des efforts d'abattement du secteur 1 . Bien qu'il ne s'agisse pas d'un pur effet externe au sens économique du terme puisque celui-ci passe par le prix du carbone, cela soulève néanmoins des questions intéressantes concernant la régulation du carbone et 
les incitations à l'abattement. Le secteur des transports en offre un très bon exemple (cf. Chakravorty et al., 2011). En effet, à partir d'une même source primaire d'énergie, le pétrole, on peut faire fonctionner soit un moteur thermique, soit produire de l'électricité pour alimenter un moteur électrique. Les émissions résultant de cette seconde utilisation pourront être capturées, mais pas les premières.

\section{Références}

Amigues J-P., Moreaux M., Schubert K. (2011). Optimal use of a polluting non-renewable resource generating both manageable and catastrophic damages. Annales d'Economie et de Statistique, 103-104, pp. 107-141.

Amigues J-P., Lafforgue G., Moreaux M. (2012-a). Optimal timing of carbon capture policies under alternative CCS cost functions. Lerna Working Paper $n^{\circ} 12.11 .368$, Toulouse School of Economics.

Amigues J-P., Lafforgue G., Moreaux M. (2012-b). Optimal timing of CCS policies with heterogeneous energy consumer sectors. Lerna Working Paper $n^{\circ} 12.13 .370$, Toulouse School of Economics. A paraître dans Environmental and Resource Economics

Ayong le Kama A., Fodha M., Lafforgue G. (2012). Optimal carbon capture and storage policies. A paraître dans Environmental Modeling and Assessement. DOI: 10.1007/s10666-012-9354-y.

Barrett S. (2009). Climate treaties with a backstop technology. Working Paper, Columbia University.

Chakravorty U., Magné B., Moreaux M. (2006). A Hotelling model with a ceiling on the stock of pollution. Journal of Economic Dynamics and Control, 30, pp. 2875-2904.

Chakravorty U., Leach A., Moreaux M. (2011). Would Hotelling Kill the Electric Car? Journal of Environmental Economics and Management, 61, pp. 2875-2904.

Coulomb R., Henriet F. (2010). Carbon price and optimal extraction of a polluting fossil fuel with restricted carbon capture. Working paper $n^{\circ} 2010-11$, Paris School of Economics.

Edmonds J., Clarke J., Dooley J., Kim S.H., Smith S.J. (2004). Stabilization of $\mathrm{CO}_{2}$ in a B2 world: insights on the roles of carbon capture and disposal, hydrogen, and transportation technologies. Energy Economics, 26, pp. 517-537.

Gerlagh R., van der Zwaan B.C. (2006). Options and instruments for a deep Cut in $\mathrm{CO}_{2}$ emissions: carbon capture or renewable, taxes or subsidies? Energy Journal, 27, pp. 2548.

Grimaud A., Lafforgue G., Magné B. (2011). Climate change mitigation options and directed technical change: A decentralized equilibrium analysis. Resource and Energy Economics, 33(4), 938-962.

Hoel M., Kverndokk S. (1996). Depletion of fossil fuels and the impacts of global warming. Resource and Energy Economics, 18, pp. 115-136. 
IEA (2006). IEA Energy Technology Essentials: $\mathrm{CO}_{2}$ Capture and Storage. Disponible sur: www.iea.org/Textbase/techno/essentials.htm.

IPCC (2005). Special Report on Carbon Dioxide Capture and Storage, Working Group III.

IPCC (2007). Climate Change 2007, Synthesis Assessment Report, Working Group III.

Islegen O., Reichelstein S. (2009). The economics of carbon capture. The Economist's Voice, December: The Berkeley Electronic Press.

Keith D., Ha-Duong M., Stolaroff J. (2006). Climate strategy with $\mathrm{CO}_{2}$ capture from the air. Climatic Change, 74, pp. 17-45.

Keith D. (2009). Why capturing $\mathrm{CO}_{2}$ from the atmosphere? Science, 325, pp. 1654-1655.

Lafforgue G., Magne B., Moreaux M. (2008-a). Energy substitutions, climate change and carbon sinks. Ecological Economics, 67, pp. 589-597.

Lafforgue G., Magne B., Moreaux M. (2008-b). The optimal sequestration policy with a ceiling on the stock of carbon in the atmosphere. In: Guesnerie, R., Tulkens, H. (Eds), The Design of Climate Policy. The MIT Press, Boston, pp. 273-304.

McKay D. (2009). Sustainable energy - Without the hot air. UIT Cambridge Ltd., Cambridge.

MIT (2007). The future of coal. Disponible sur: http://web.mit.edu/coal.

Toman M.A., Withagen C. (2000). Accumulative pollution, clean technology and policy design. Resource and Energy Economics, 22, pp. 367-384. 